\title{
14-3-3 Proteins Are Part of an Abscisic Acid-VIVIPAROUS1 (VP1) Response Complex in the Em Promoter and Interact with VP1 and EmBP1
}

\author{
Thomas F. Schultz, ${ }^{1}$ J oaquin Medina, ${ }^{2}$ Alison Hill, and Ralph S. Quatrano ${ }^{3}$ \\ Department of Biology and Curriculum in Genetics and Molecular Biology, University of North Carolina at Chapel Hill, \\ Chapel Hill, North Carolina 27599-3280
}

Protein-DNA complexes were formed when nuclear extracts from embryogenic rice suspension cultures or maize embryos were incubated with an abscisic acid-VIVIPAROUS1 (VP1) response element (Emla) from the Em promoter. Monoclonal antibodies generated to GF14, a 14-3-3 protein from plants, resulted in gel retardation of the Em1a-protein complexes. Antibodies generated to the C and N termini of GF14 detected protein isoforms in rice nuclear and cytoplasmic extracts, but no differences in distribution of the GF14 isoforms were recognized between the nucleus and cytoplasm or when abscisic acid-treated and untreated tissues were compared. When recombinant GF14 fusion proteins from rice were added to nuclear extracts, novel complexes were formed that required the dimerization domain of GF14. Chemical cross-linking showed that GF-14 interacted with the basic leucine zipper factor EmBP1, which binds specifically to Emla, and with VP1, which transactivates Em through Emla. GF14 proteins from rice were shown to interact with VP1 in yeast through the dimerization domain of GF14. Our results indicated that GF14 interacts with both site-specific DNA binding proteins (i.e., EmBP1) and tissue-specific regulatory factors (i.e., VP1) and may provide a structural link between VP1 and the Emla transcriptional complex.

\section{INTRODUCTION}

We have used the Em gene as a model to understand the molecular basis of how abscisic acid (ABA) regulates embryo-specific gene expression. In whole plants, Em expression is limited to maturing embryos; Em transcripts normally fail to accumulate in precociously germinated seeds or in response to $A B A$ in vegetative tissues. The viviparous phenotype of maize (i.e., embryos that germinate while still attached to the ear) has provided an important framework on which to study the molecular mechanisms involved in the regulation of Em. viviparous (vp) mutants are characterized in part by their reduced levels of $A B A$ or sensitivity to endogenous ABA (Zeevart and Creelman, 1988; Rock and Quatrano, 1994, 1995; McCarty, 1995). Em transcripts do not accumulate in vp mutants that are ABA deficient (e.g., vp5) or have normal levels of ABA but are ABA insensitive (e.g., vp1) (McCarty et al., 1991). Similar mutations have been identified in other plants, but the most studied is the vp1 equivalent in Arabidopsis, namely, the abscisic acid-insensitive 3

\footnotetext{
${ }^{1}$ Current address: School of Botany, University of Melbourne, Parkville 3052, Victoria, Australia.

${ }^{2}$ Current address: Departamento de Mejora Genetica y Biotecnologia, CIT-INIA, Carretera de La Coruña, Km 7.5-28040, Madrid, Spain.

3 To whom correspondence should be addressed at Department of Biology, University of North Carolina, Chapel Hill, NC 27599-3280. E-mail rsq@unc.edu; fax 919-962-6840.
}

(abi3) mutant (Nambara et al., 1992; Parcy et al., 1994). In abi3 seeds, Em transcripts are also absent (Finkelstein, 1993; Parcy et al., 1994). Ectopic expression of $A B I 3$ in vegetative tissue of Arabidopsis seedlings, in the presence of $A B A$, results in the expression of the AtEml gene (Parcy et al., 1994). These results demonstrate clearly that Em expression requires both $A B A$ and either $V P 1$ or $A B I 3$ and that its tissue-specific regulation appears to be under the direct control of VP 1 or ABI3.

By use of both a transient assay and transgenic plants, cis elements within the Em promoter have been identified that can confer ABA-inducible expression to a reporter gene (Marcotte et al., 1988, 1989). Both the Vpl and Abi3 genes have been cloned as well as their homologs from rice, oat, and bean (McCarty et al., 1989b; Giraudat et al., 1992; Hattori et al., 1994; Bobb et al., 1995; J ones et al., 1997). Sequence comparisons of all five homologs indicate that three regions have highly conserved amino acid residues (Bobb et al., 1995; Hill et al., 1996; J ones et al., 1997). When VP1 is overexpressed in transient assays, it transactivates the Em promoter in the absence of exogenous $A B A$ and causes a synergistic effect in the presence of added ABA (McCarty et al., 1991; Hattori et al., 1995; Vasil et al., 1995). Both the $\mathrm{N}$-terminal transactivation domain (McCarty et al., 1991) and a conserved basic domain (BR2) (Hill et al., 1996) of VP 1 are required for VP 1 transactivation of $\mathrm{Em}$ in transient assays. 
The same cis elements in the Em promoter that respond to ABA (i.e., Emla) also mediate transactivation by VP1 (Vasil et al., 1995). Thus, $A B A$ and VP1 activate Em expression through Emla, the ABA-VP1 response element within the Em promoter.

Evidence gained from work in other systems shows that protein-protein interactions at the site of response elements are critical for selected activation of gene expression (reviewed in Goodrich et al., 1996). A basic leucine zipper (bZIP) transcription factor (EmBP1) has been identified that can bind to the ABA-responsive elements (Emla and Emlb) within the Em promoter (Guiltinan et al., 1990). Recently, it has been shown that a conserved region near the $C$ terminus of VP1 (BR3) exhibits sequence-specific DNA binding activity for Sph elements that are present in several plant promoters, including Em (Suzuki et al., 1997). Although this observation is a significant step forward in our understanding of the function of VP 1 , it does not fully explain its role in regulating Em. For example, VP1 can transactivate the Em promoter through $\mathrm{G}$ box elements in the absence of Sph sequences (Vasil et al., 1995). Also, the vp1-McW allele truncates the $C$ terminus of VP1, removing its DNA binding domain; yet, this mutant still undergoes seed maturation and accumulates Em transcripts (McCarty et al., 1989a). Furthermore, when VP1 lacks only its conserved BR2 domain, with the activation and $B R 3$ regions intact, transactivation of Em in transient assays has not been observed (Hill et al., 1996). Hence, the site-specific DNA binding domain of VP1 is not required or sufficient for all of its functions (McCarty et al., 1989a; Suzuki et al., 1997).

In vitro, a truncated VP1 that lacks the activation domain and a portion of BR3 can dramatically enhance the ability of EmBP1 to bind to Emla (Hill et al., 1996) as well as stimulate transcription specifically from the Em promoter in HeLa nuclear extracts (Razik and Quatrano, 1997). A smaller, 40amino acid region of VP1 that contains the BR2 fragment does bind weakly and nonspecifically to DNA, as demonstrated by UV cross-linking (Hill et al., 1996). Also, when this BR2-containing fragment is fused with LacZ, it confers on LacZ the ability to enhance EmBP binding to Emla. These in vitro results further support the conclusion that the function of VP 1 is modular in nature and that the region that includes $B R 2$ is critical for Em expression.

We began this study to identify proteins that interact with Emla and may structurally link VP1 to this region by protein-protein interactions. Because plant 14-3-3 proteins were initially identified in association with $\mathrm{G}$ box elements (like Emla) in promoters (like Em) that are responsive to various signal transduction pathways (de Vetten et al., 1992; Lu et al., 1992; Chen et al., 1994; Ferl, 1996), we asked whether these 14-3-3 or GF14 proteins (Lu et al., 1992) could be found in association with Emla, the ABA-VP1 response element. We present evidence that a GF14 epitope is found in the transcriptional complex associated with Emla. Furthermore, when recombinant GF14 proteins were added to nuclear extracts, Emla-specific complexes were formed that contained the added GF14 proteins. Because GF14 proteins do not bind DNA, they are apparently assembled into proteinDNA complexes through their interaction with Emla-specific DNA binding proteins. We show by chemical cross-linking that these same GF14 recombinant proteins from rice bind to EmBP1 as well as VP1. GF14 also interacted specifically with VP1 in the yeast two-hybrid system. Assembly of GF14 into an Emla transcriptional complex in nuclear extracts as well as interaction with VP 1 in yeast required the dimerization domain of GF14. Hence, dimers of GF14 may provide a structural link between VP1, EmBP1, and the ABA-VP1 response complex in the Em promoter.

\section{RESULTS}

\section{Monoclonal Antibodies Generated to GF14 Proteins Recognize an Epitope in the ABA-VP1 Response Complex Emla}

Several groups reported previously that plant GF14 proteins are complexed with $\mathrm{G}$ box binding factors that recognize cis elements in promoters responsive to various signals (de Vetten et al., 1992; Lu et al., 1992; Chen et al., 1994). To test whether these same GF14 antibodies are able to recognize GF14 proteins in G box binding complexes in the Em promoter (i.e., Emla), electrophoretic mobility shift assays (EMSAs) were performed in the presence of monoclonal antibodies (MAb) that recognized epitopes at the C-terminal region of GF14 (MAb GF14 [15] and MAb GF14 [19]; Lu et al., 1994) and a polyclonal antibody (Ab) raised against a peptide from a conserved region in the dimerization domain near the N terminus of GF14 proteins (FCA; Marra et al., 1994). Nuclear extracts were prepared from untreated or ABA-treated $(100 \mu \mathrm{M})$ embryogenic rice suspension cells and incubated with either the Emla or AT-rich (ATR) probes from the Em promoter (Guiltinan et al., 1990; Hill et al., 1996). Protein factors present in nuclear extracts recognized the Emla element and formed protein-DNA complexes detected in EMSA experiments (Figure 1). We also observed an increase in binding activity to the Emla probe in extracts from ABA-treated tissue, as was previously reported (Guiltinan et al., 1990). When these nuclear extracts were incubated with MAb GF14(15), the migration of the Emla complexes was retarded further (supershift), indicating the presence of a GF14 epitope in these complexes. A second monoclonal antibody, MAb GF14(19), also resulted in a supershift (data not shown). Interestingly, the polyclonal antibody AbFcA was not able to supershift these complexes (data not shown), even though it recognized proteins in nuclear extracts (see below).

No major differences in EMSAs were observed using the two monoclonal antibodies on extracts prepared from untreated and ABA-treated cells (Figure 1 and data not shown). The observed supershift of complexes formed with the mono- 


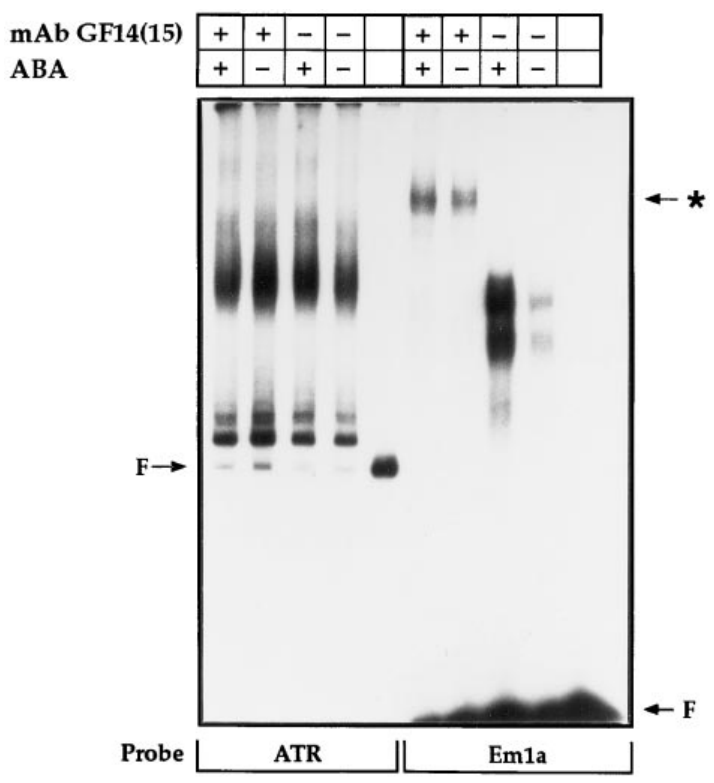

Figure 1. Antibody Recognition of a GF14 Epitope in Emla Complexes Formed in Nuclear Extracts from Rice Cells.

Nuclear extracts were prepared from embryogenic rice suspension cells that were either ABA treated $(100 \mathrm{mM})(+)$ or untreated $(-)$. The DNA binding reactions contained nuclear extract $(1 \mu \mathrm{g}$ of protein) and $1 \mu \mathrm{g}$ of poly $(\mathrm{dl}-\mathrm{dC})$ in the presence $(+)$ or absence $(-)$ of the GF14 monoclonal antibody MAb GF14(15), as indicated. Radiolabeled Emla or ATR probes from the Em promoter were added to these reactions and analyzed by EMSA. The asterisk indicates antibody-Emla binding complexes. $F$, free probe.

clonal antibodies occurs only with the Emla probe and not with complexes formed with the ATR probe (Figure 1). EMSAs with Emla were also performed on nuclear extracts prepared from maize wild-type and vp1 embryos isolated 20 days after pollination. The addition of MAb GF14(19) resulted in a supershift of the Emla binding complex in both wild-type and vp1 mutant extracts (Figure 2).

Hence, it appears that monoclonal antibodies that recognize epitopes near the C-terminal regions of GF14 proteins detected GF14 proteins that are complexed with Emla in maize and rice nuclear extracts. Extracts prepared from tissue treated with ABA or lacking VP1 also showed the same association of GF14 epitopes in the Emla complex.

\section{Incorporation of Maltose Binding Protein-GF14 Fusion Proteins into Emla Complexes}

A different approach was taken to demonstrate a specific interaction between GF14 proteins and the Emla element. We added recombinant GF14 proteins fused to the maltose binding protein (MBP) to nuclear extracts and monitored their assembly into DNA complexes with antibodies generated against MBP. Using a rice GF14 CDNA (E10, see below) as a probe, we screened a cDNA library from rice suspension cultures at low stringency and characterized four rice cDNAs that encode GF14 proteins (osGF14). Alignment of GF14 amino acid sequences from rice, Arabidopsis, tobacco, and maize with a 14-3-3 protein from sheep (Figure 3 ) demonstrates the high degree of sequence conservation present among members of this gene family.

MBP-osGF14a and MBP-osGF14b were prepared, added to nuclear extracts, and subjected to EMSAs. Increasing amounts of MBP-osGF14a added to nuclear extracts resulted in the appearance of an additional Emla binding complex with a slower mobility on EMSA (Figures 4A and $4 B$, indicated by asterisks). When an anti-MBP antibody was added to nuclear extracts containing MBP-osGF14a, this new complex was not detected (Figure $4 A$ ). With longer exposures, a supershift was observed (data not shown). In the absence of nuclear extracts, MBP-osGF14a was not capable of interacting with Emla (data not shown). When MBP-LacZ was added to nuclear extracts, no additional complexes were formed (Figure 4A, second lane), indicating specificity

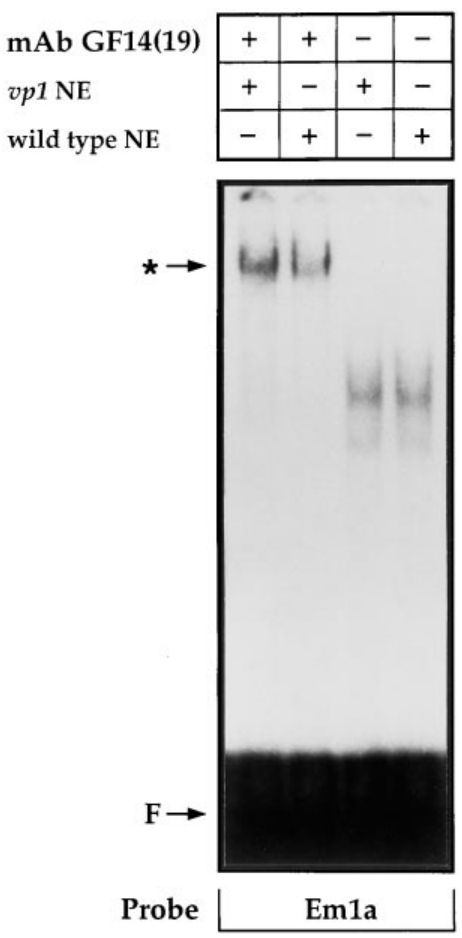

Figure 2. Antibody Recognition of a GF14 Epitope in Emla Complexes from Maize Wild-Type and vp1 Nuclear Extracts.

Nuclear extracts (NE) (1 $\mu \mathrm{g}$ of protein) were prepared from maize wild-type and vpl embryos (20 days after pollination), and an EMSA was performed as described in Figure 1 . The asterisk indicates antibody-Emla binding complexes. F, free probe. 


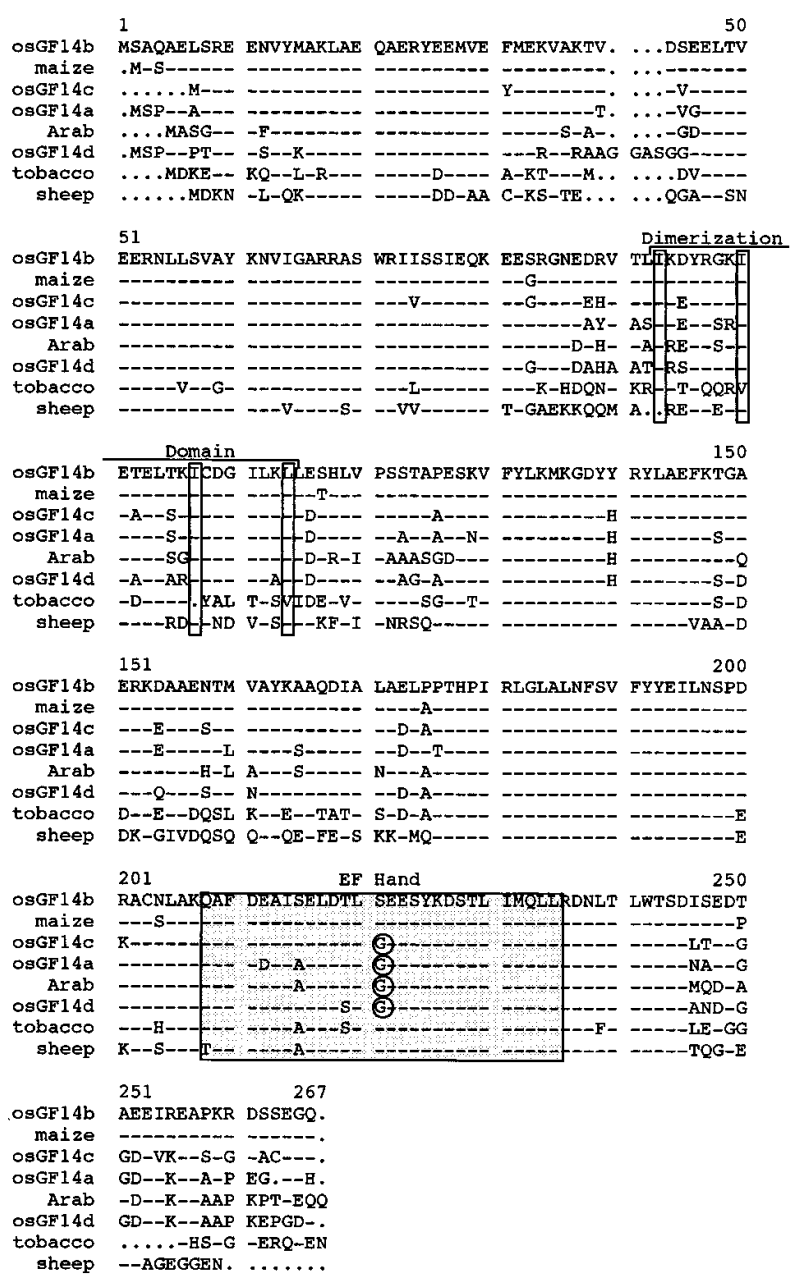

Figure 3. Alignment of GF14 Amino Acid Sequences Deduced from the Four Rice Clones (osGF14a, osGF14b, osGF14c, and osGF14d) and Maize, Arabidopsis, Tobacco, and Mammalian (Sheep) 14-3-3 Proteins.

Standard single-letter codes were used for each amino acid. Dashes represent identical amino acids, and periods represent gaps inserted into the sequences for optimal alignment. The sequence including amino acids 93 to 114 represents the dimerization domain, with the boxed amino acids forming the heptad leucine/isoleucine repeat. The shaded box (amino acids 208 to 235) marks the putative EF hand domain, with the critical central glycines designated by circles. Arab, Arabidopsis.

for the GF14 portion of the fusion. The addition of MBPosGF14b(II) to nuclear extracts also resulted in the formation of a new complex on EMSA (Figure 4B). However, osGF14b(I), which lacks 101 amino acids from the $\mathrm{N}$ terminus, did not form additional nuclear complexes (Figure 4C). These data show that the addition of MBP-GF14 fusion proteins to nuclear extracts results in the incorporation of these proteins into protein-Emla complexes and a requirement for the $\mathrm{N}$-terminal dimerization region for the complex to form.

\section{Rice GF14 Protein Localization and Expression}

Both monoclonal and polyclonal antibodies generated to GF14 detected a doublet at $\sim 30 \mathrm{kD}$ on protein gel blots in both cytoplasmic and nuclear extracts from embryogenic rice suspension cells (Figure 5A). No differences were observed between untreated sample or samples treated with ABA (Figure 5A). The location of the bands on these protein gel blots is consistent with the predicted molecular masses from analysis of the cDNA sequences. The AbFcA antibody detected the same doublet in both nuclear and cytoplasmic extracts, whereas the lower band of the doublet detected by MAb GF14(19) appeared to differ between the cytoplasmic and nuclear extracts. These same antibodies were then tested on MBP-osGF14 fusion proteins (Figure 5B). AntiMBP antibodies showed that equal quantities of fusion protein were loaded in each lane. AbFcA reacted with both MBP-osGF14a and MBP-0sGF14b equally, whereas the MAb GF14(19) reacted much more strongly with MBPosGF14b than with MBP-osGF14a, indicating specificity between different GF14 family members in their ability to bind this monoclonal antibody.

\section{Rice GF14 Proteins Interact with EmBP1 and VP1}

The presence of GF14 in the ABA-VP1 response complex Emla suggested that it may be interacting directly with VP 1 and EmBP1, a bZIP protein that binds the Em1 response elements. To determine whether there is an interaction between GF14 and VP1, and possibly to identify other proteins that interact with VP1, we performed a two-hybrid screen with yeast. A cDNA encoding a truncated VP1 that contained the required domains for Em expression (amino acids 190 to 588) was translationally fused to the LexA DNA binding domain and used as bait in yeast to screen a cDNA library from embryogenic rice suspension cells. From these colonies, a number of different clones were identified. One clone (E10) was 821 bp long and contained a 477-bp open reading frame encoding a protein with high homology to plant GF14 proteins (Lu et al., 1992). Using E10 as a probe, we isolated and characterized several osGF14 clones (Figure 3 ) that were then tested for their ability to interact with VP 1 in yeast.

The yeast phenotypes resulting from interaction between the VP1 bait and the osGF14 proteins are shown in Figure 6. Yeast (EGY48) were transformed with either the interactor vector alone with no insert (Vector) or with three different rice GF14 proteins (osGF14a, osGF14b, and osGF14c) cloned into the interactor vector. These yeast were cotransformed with either the VP1 bait or with a nonspecific bait (Control), as described in Methods. In the presence of leu- 
A

B

C

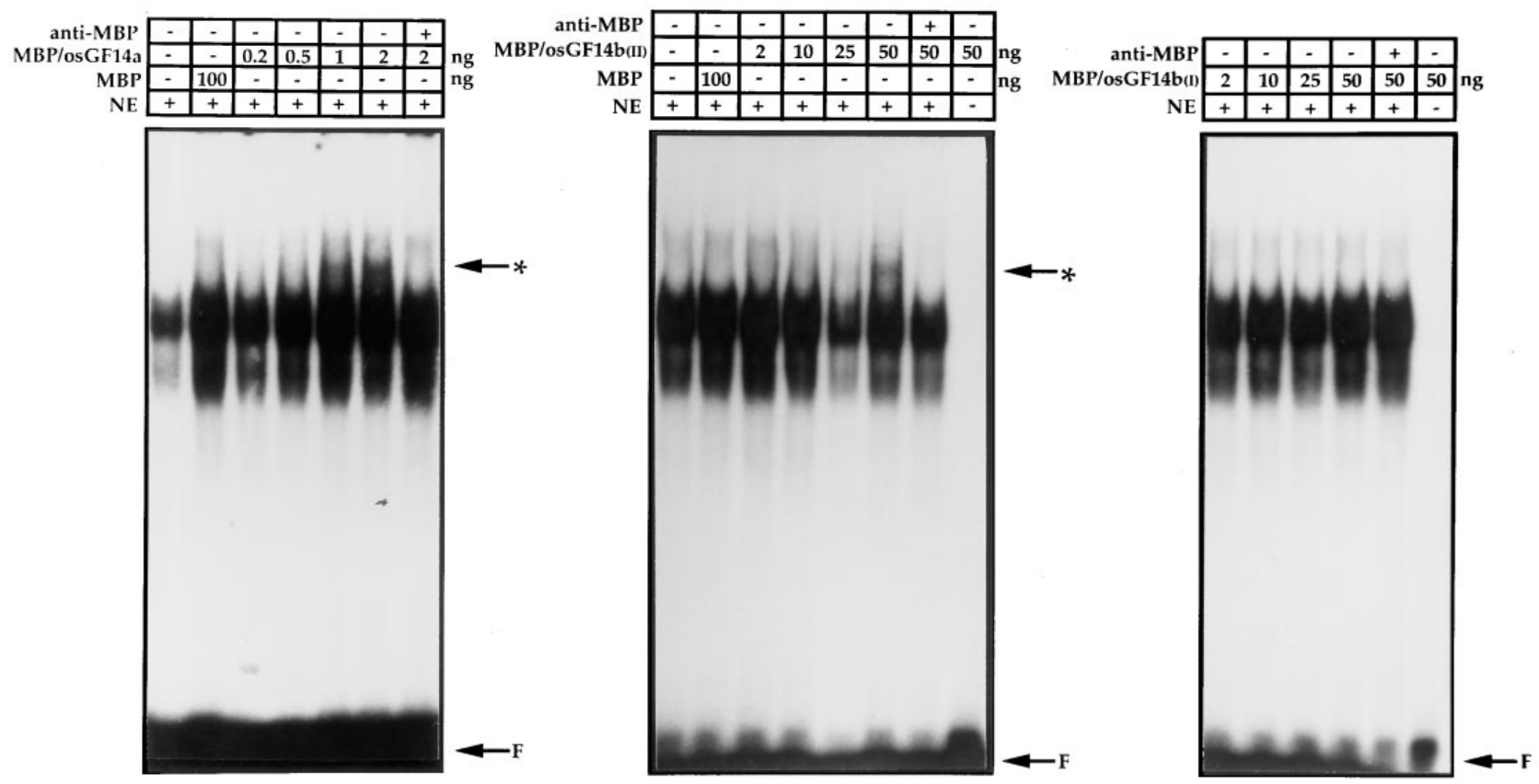

Figure 4. MBP-osGF14 Fusion Proteins Are Incorporated into Protein-Emla Complexes Formed in Nuclear Extracts from Rice Cells.

(A) Nuclear extracts $(\mathrm{NE})$ were prepared from ABA-treated $(100 \mu \mathrm{M})$ cells, and an EMSA was performed as described in Figure 1. The MBPosGF14a recombinant protein (0.2 to $2 \mathrm{ng}$ of protein) or $100 \mathrm{ng}$ of free MBP was added to nuclear extracts in the presence $(+)$ or absence $(-)$ of the anti-MBP antibody. The asterisk indicates the new Emla binding complex formed in extracts containing MBP-osGF14a.

(B) Same as given in (A), except that 2 to $50 \mathrm{ng}$ of the MBP-osGF14b(II) (amino acids 8 to 261) recombinant protein was added to nuclear extracts. The asterisk indicates the new Emla binding complex formed in extracts containing MBP-osGF14a.

(C) Same as given in (A), except that 2 to $50 \mathrm{ng}$ of the MBP-osGF14b(I) (amino acids 102 to 261) recombinant protein was added to nuclearextracts. $F$, free Emla probe.

cine, each of the transformants demonstrated growth (Figure 6 , top), whereas in the absence of leucine, only cells grown on galactose transformed with VP 1 bait and osGF14 interactors were able to grow (Figure 6 , bottom right). Also, no interaction was detected between these osGF14 proteins and two other nonspecific baits (data not shown).

Table 1 shows quantitative $\beta$-galactosidase assays and indicates that full-length osGF14a and osGF14C and a truncated osGF14b(I) (amino acids 102 to 261) resulted in the same 20 -fold increase in $\beta$-galactosidase activity over the control vector. However, the osGF14b(II) (amino acids 8 to 261) interactor resulted in an additional 10-fold increase over osGF14a, osGF14C, and osGF14b(I) and an increase of $>200$-fold compared with the control vector. This result not only indicates differences between GF14 proteins (compare osGF14a and osGF14c with osGF14b[II]) but suggests that the $\mathrm{N}$ terminus of osGF14b (compare osGF14b[I], which lacks the N-terminal 101 amino acids, with osGF14b[II]) is important for the interaction with VP1.
To determine whether there is physical interaction between GF14 and EmBP1 as well as to confirm the interaction between GF14 and VP1 in yeast, we performed protein-protein cross-linking experiments with MBP fusion proteins. We incubated MBP-osGF14b(I) with either MBP-VP1, MBP-EmBP1, or MBP-cl28 (a negative control; see Methods) in the presence of the cross-linking reagent dimethyl 3,3'-dithiobispropionimidate. After cross-linking, the proteins that were bound to MBP-osGF14b(I) were immunoprecipitated with MAb GF14(19), separated on SDS-acrylamide gels, blotted, and probed with antibodies generated against cl28, VP1, and GF14. As shown in Figure 7, both MBP-VP1 and MBPEmBP1 coimmunoprecipitated with MBP-osGF14b(I), but $M B P-c 128$, the negative control, did not. These results confirmed the yeast data, that is, that VP1 and GF14 proteins interact, and extend our observations to include the result that a GF14 protein is able to bind to EmBP1, a specific bZIP protein that recognizes the $A B A-V P 1$ response element (Emla) in the Em promoter (Guiltinan et al., 1990). 
A

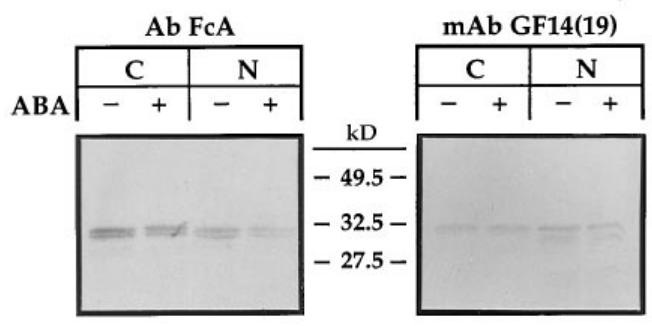

B

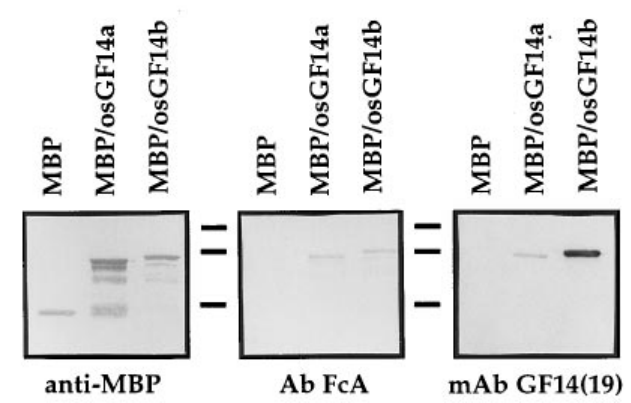

Figure 5. Protein Gel Blot Analysis of Extracts from Rice Cells and Recombinant Fusion Proteins.

(A) Cytoplasmic (C) and nuclear (N) extracts prepared from embryogenic rice suspension cells, either ABA treated $(100 \mathrm{mM})(+)$ or untreated $(-)$, were separated on $12 \%$ SDS-polyacrylamide gels and blotted onto nitrocellulose. GF14 proteins were detected with a polyclonal GF14 antibody (Ab FCA) or a monoclonal GF14 antibody (mAb GF14[19]). Protein molecular mass markers in kilodaltons (kD) are indicated between the protein gel blots.

(B) Free MBP and purified MBP fusions of osGF14a or osGF14b (200 ng of protein each) were separated on 10\% SDS-polyacrylamide gels and blotted to nitrocellulose. Separate protein gel blots were probed with the anti-MBP antibody and two GF14 antibodies as in (A). Molecular mass markers shown between each protein gel blot are 101,83 , and $50.6 \mathrm{kD}$, from top to bottom, respectively.

\section{DISCUSSION}

We characterized three novel rice GF14 amino acid sequences that share 80 to $90 \%$ identity when compared with each other and one previously isolated from rice (Kidou et al., 1993). They are all members of the non- $\epsilon$ group (III) of 14-3-3 proteins, sharing 70 to $90 \%$ identity with amino acid sequences from this group, which comprises $>80 \%$ of the plant GF14 proteins (Wu et al., 1997). Interestingly, the osGF14b cDNA shares unusually high $(98 \%)$ amino acid identity with a GF14 amino acid sequence from maize (de Vetten et al., 1992), indicating that these two GF14 proteins may be functional homologs. GF14 proteins are characterized by their dimerization domain and an EF hand motif (Lu et al., 1994), both of which are highly conserved in all 14-3-3 proteins (Figure 3). A central glycine residue of the EF hand motif is critical for calcium binding activity (Strynadka and J ames, 1989). Analysis of 26 plant GF14 amino acid sequences (10 from Arabidopsis) showed that eight plant proteins have a substitution for the conserved glycine at this position (usually serine), whereas all of the others have the conserved glycine. os GF 14b, isolated in this study, is one of the plant GF14s that lack the conserved glycine, along with all members of the $\epsilon$ subgroup (II), four of which are from plants (Wu et al., 1997). This observation points to a potential difference in the ability to bind calcium among members of the GF14 family of proteins. The idea that different isoforms of GF14 proteins may have specific functions during plant development is also supported by the results of Daugherty et al. (1996), who demonstrated cell-specific expression of GF14 chi from Arabidopsis.

Antibodies to different regions of the GF14 protein detected some interesting differences in cellular distribution and in reaction to different rice isoforms. Monoclonal (MAb GF14[19]) and polyclonal (AbFCA) antibodies raised against the $\mathrm{C}$ and $\mathrm{N}$ termini of GF14, respectively, both detected a doublet of $\sim 30 \mathrm{kD}$ in cytoplasmic and nuclear extracts from embryogenic rice suspension cells. No differences were observed between extracts from untreated samples or from ABA-treated samples with either antibody. Interestingly, MAb GF14(19) detected differences in the mobility of the lower band of the doublet between cytoplasmic and nuclear extracts and reacted much more strongly with the fusion protein MBP-0sGF14b than with MBP-osGF14a. AbFcA reacted identically with nuclear and cytoplasmic extracts and with both MBP-osGF14a and MBP-osGF14b. Because the monoclonal antibody recognizes an epitope in the highly variable C terminus (Lu et al., 1994; Ferl, 1996), it appears then that certain isoforms may be partitioned differently between the nucleus and cytoplasm. The peptide in the $\mathrm{N}$-terminal region used to generate $\mathrm{AbFcA}$ is quite conserved (Marra et al., 1994) and does not recognize such differences. Monoclonal antibodies raised against each GF14 isoform will be valuable in determining more specifically whether there are differences in the localization and function of the various isoforms.

Our results given above with antibodies generated against both the $\mathrm{C}$ and $\mathrm{N}$ termini indicate that GF14 proteins are found in the nucleus, confirming previous work that plant GF14 proteins are complexed with transcription factors bound to DNA, even though they themselves are unable to bind DNA directly (de Vetten et al., 1992; Lu et al., 1992; Chen et al., 1994). The addition of antibodies that recognize the C-terminal domain of GF14 resulted in a supershift of protein-Emla complexes on EMSA. The supershift was not observed with protein-DNA complexes formed on a different DNA element (ATR tract) present in the Em promoter, indicating specificity of interaction between Emla binding factors and GF14. These results demonstrate the presence of a GF14 epitope in Emla complexes, similar to nuclear 

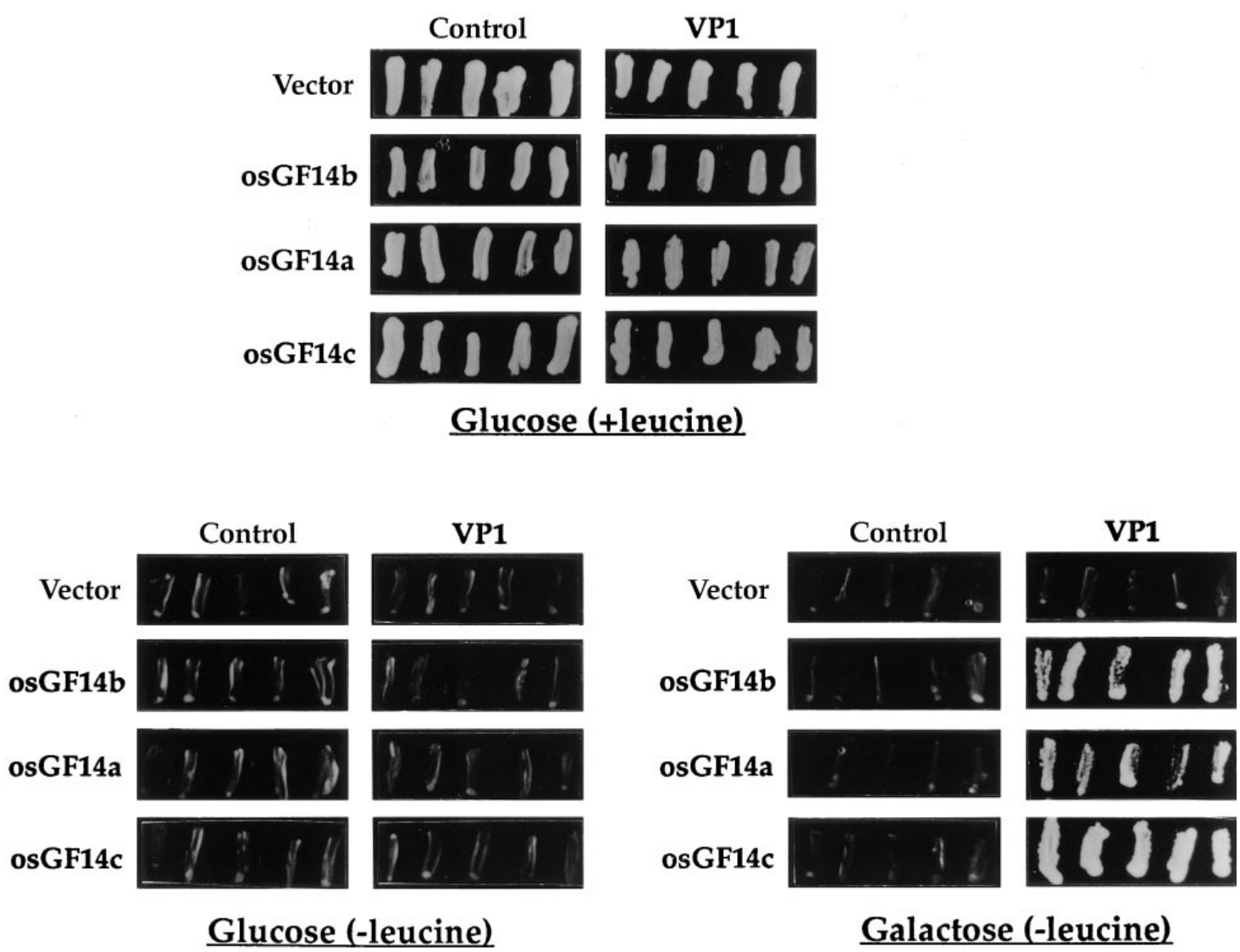

Galactose (-leucine)

Figure 6. Growth of Yeast Containing VP1 as Bait and Rice GF14 Interactors (osGF14a, osGF14b, and osGF14c) on Different Media.

Yeast (EGY48), containing VP1 or a nonspecific bait (Control), was cotransformed with either an empty interactor vector (Vector) or with three different 0sGF14s (see Methods). Transformants were transferred to plates with (+) or without (-) leucine and containing either glucose or galactose/raffinose in the medium. The growth phenotypes of five independent colonies are shown for each transformation.

complexes bound to an Emla-like element (i.e., G box) in the alcohol dehydrogenase and osmotin promoters. Observations of a supershift in the EMSA experiments with the Emla probe and antibody to the C terminus of GF14 (MAb GF14[19]) suggest that the C-terminal domain is exposed in the Emla complex. Under the same conditions, no supershift was observed with $A b F c A$, an antibody raised against a peptide in the $\mathrm{N}$-terminal region of 14-3-3 proteins. Based on these results, it is tempting to speculate that the $\mathrm{N}$ terminus (i.e., dimerization domain) is buried in the Emla complex. However, these results could be explained by AbFcA not recognizing the native protein in the EMSA experiments. Also, the MBP-GF14 fusion protein is recognized in the Emla complex by the antibody generated against MBP, even though the MBP is linked at the N terminus of GF14.

To confirm more directly the presence of GF14 in the complex, to demonstrate a possible role of GF14 in assembling the complex, and to determine the domains involved, we showed that the addition of two different bacterially
Table 1. Quantitative $\beta$-Galactosidase Expression in Yeast Cultures Containing VP1 as Bait and Individual osGF14 Interactors

\begin{tabular}{lll}
\hline Interactor & $\begin{array}{l}\text { Growth } \\
\text { (Galactose-Leucine) }\end{array}$ & $\begin{array}{l}\text { Galactosidase Activityc } \\
\text { (Units) }\end{array}$ \\
\hline Vector & - & $0.37 \pm 0.12$ \\
osGF14b(I) & + & $7.8 \pm 1.70$ \\
osGF14b(II) & + & $83.7 \pm 34.1$ \\
oSGF14a & + & $8.1 \pm 4.1$ \\
osGF14c & + & $7.0 \pm 1.8$ \\
\hline
\end{tabular}

a Empty interactor vector (Vector), osGF14b(I) (amino acids 102 to 261), osGF14b(II) (amino acids 8 to 261), osGF14a (amino acids 1 to 259), and osGF14c (amino acids 25 to 261).

$\mathrm{b}(+)$ indicates growth; $(-)$ indicates no growth.

${ }^{c}$ Cultures from five independent transformants were assayed, and the average units are given with standard deviations. 


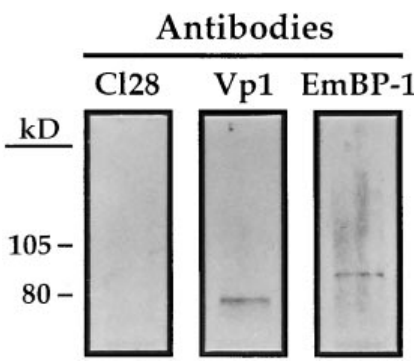

Figure 7. Cross-Linking of VP1 and GF14 Proteins in Vitro.

VP1 and OSGF14b were expressed and purified from bacteria as MBP fusions. MBP-osGF14b(I) ( 60 kD) was mixed with MBP-VP1 $(\sim 80 \mathrm{kD}), \mathrm{MBP}-\mathrm{EmBP} 1(\sim 95 \mathrm{kD})$, or MBP-cl28 ( 80 kD) in the presence of the cross-linking reagent dimethyl 3,3'-dithiobispropionimidate. The cross-linked proteins were then immunoprecipitated with MAb GF14(19), separated on 10\% SDS-polyacrylamide gels, and detected by using protein gel blot analysis with specific antibodies, as indicated. The Vpl and EmBP1 antibodies detected the presence of their respective MBP fusion proteins in an antibody complex with MBP-osGF14b(I), but the antibody to $\mathrm{Cl} 28$ did not recognize the $\mathrm{cl} 28$ fusion in a cross-linked complex with osGF14. Numbers at left indicate protein molecular mass markers in kilodaltons (kD).

expressed MBP-GF14 fusion proteins to nuclear extracts resulted in the formation of novel Emla-protein complexes in EMSA experiments. Antibodies generated against MBP specifically abolished these new complexes in EMSA experiments, indicating that the fusion proteins were indeed in the Emla complex. Neither free MBP nor a truncated MBPGF14 lacking 101 amino acids at the $N$ terminus became incorporated into these protein-DNA complexes. Because the same GF14 fusion proteins were unable to bind Emla directly, these results demonstrate the ability of GF14 to interact with and form a complex (through its dimerization domain) with a transcription factor specific for the Emla element.

One possible (but not exclusive) type of transcription factor would be the bZIP class, for example, EmBP1, which has been shown to bind specifically to $G$ boxes such as Emla (Izawa et al., 1993; Nantel and Quatrano, 1996). In fact, we showed in this study that MAb GF14(19) coimmunoprecipitated the MBP fusion proteins EmBP1 and osGF14b after cross-linking. This bZIP class of transcription factors is also interesting in that it forms dimers through a domain that is similar in structure to the dimerization domain of GF14, namely, leucine/isoleucine heptad repeat (Schindler et al., 1992; Lu et al., 1994). B ecause GF14 and G box binding factors have been reported to interact in earlier studies (Ferl, 1996), it would be interesting to determine whether heterodimers might form between bZIP and GF14 family members (see de Vetten et al., 1992), and if so, what effect this would have on binding specificities (see Nantel and Quatrano, 1996). Regardless, one can extend the biochemical approach described here, that is, of adding recombinant GF14 fusion proteins to nuclear extracts, to cell-free transcription reactions by using templates from plants (Razik and Quatrano, 1997). In this way, one can use such nuclear extracts not only to identify the nuclear factor(s) that specifically binds Emla and interacts with GF14 but also to identify any component(s) that is required for this complex to form and affect transcription.

Because the ABA-inducible Em gene requires VP1 (or its homolog in Arabidopsis, ABI3) for tissue-specific expression (McCarty et al., 1991; Finkelstein, 1993; Parcy et al., 1994), we used both immunoprecipitation and the yeast two-hybrid system to show physical interaction between VP1 and GF14. Using an internal fragment (amino acids 190 to 588) of VP 1 as bait in a yeast two-hybrid screen, one interactive clone isolated from a rice cDNA library prepared from embryogenic rice suspension cells encoded a GF14 protein. To further determine whether there were any functional differences among the family of rice GF14 proteins that we characterized, we tested full-length as well as truncated clones for the ability of their products to interact with VP1 in yeast. Although all three rice clones interacted with VP1, quantitative differences were observed in the interaction between different rice GF14s and VP1. For example, osGF14b interacted 10 times more strongly than did either osGF14a or osGF14c. In addition, we found that osGF14b interacted 10 times more strongly than a truncated osGF14b, which lacks the $\mathrm{N}$-terminal 101 amino acids. This deletion removes a portion of the dimerization domain (see Figure 3). Although this interaction is not as strong as some involving plant proteins in yeast (e.g., Schultz and Quatrano, 1997), the level is clearly significant when compared with the ranges observed (Estojak et al., 1995).

Because the $\mathrm{N}$-terminal dimerization domain is required both for Emla complex formation and interaction with VP1 in yeast, we reasoned that VP1 might be required to form Emla-GF14 complexes through its interaction with the $\mathrm{N}$ terminus of GF14. However, we showed in this study that nuclear extracts from embryos of vp 1 mutants were able to form Emla complexes, indicating that the lack of VP1 protein in mutant tissue did not prevent the association of GF14 with Emla in vitro.

Although the crystal structure of $14-3-3$ is known (Liu et al., 1995), the exact function(s) of this family of related proteins is not clear at present. However, considerable evidence exists to support their role as mediators of protein-protein interactions (i.e., a chaperone or scaffolding function) that are involved in kinase-related events in signaling pathways (Ferl, 1996) and in enzyme function (Huber et al., 1996; J ahn et al., 1997). Interaction of 14-3-3s with signaling proteins appears to be mediated in many cases by the recognition of a phosphoserine binding motif in the C-terminal domain, initially identified in Raf1. Muslin et al. (1996) recently identified a common phosphoserine binding site on many proteins that interact with 14-3-3 and showed that peptides containing this motif could disrupt 14-3-3 complexes and interfere with the activation of oocyte maturation by Raf1. A sequence 
of amino acids in the maize VP1 bait that interacts with osGF14, that is, amino acids 430 to 436 (RSTHSGP), is similar to the consensus 14-3-3 binding motif (RSXS*XP) identified by Muslin et al. (1996) and present in the plant enzyme nitrate reductase, which also interacts with $14-3-3$ protein (Huber et al., 1996). Uncharacterized motifs for 14-3-3 binding are known to exist, however (Aitken, 1996). Interestingly, the conserved 14-3-3 binding site is found only in the VP1 homologs of the monocots rice (Hattori et al., 1994) and oat (J ones et al., 1997) but not in the VP 1 homologs from the dicots Arabidopsis (Giraudat et al., 1992) and bean (Bobb et al., 1995). Also, the interaction between 14-3-3 and $\mathrm{H}^{+}$-ATPase in Arabidopsis appears not to involve this conserved site (J ahn et al., 1997). Furthermore, we showed in this study that the region of GF14 required for its incorporation into the Emla complex and interaction with VP1 (i.e., N-terminal dimerization site) lacks the consensus 14-3-3 binding site. Whether the conserved 14-3-3 binding motif or some other GF14 binding site of maize VP1 plays any role in the transactivation of Em is not yet known.

It is also clear that the ABA response pathway includes changes in the level of phosphorylation of undefined substrates. For example, a phosphatase activity associated with the abi1 locus in Arabidopsis appears to be required for certain ABA responses (Giraudat et al., 1994; Leung et al., 1994, 1997; Meyer et al., 1994; Pei et al., 1997). Also, ABA was shown to induce in aleurone cells a rapid and transient activation of a MAP kinase, which is required for the activation of rab16 gene expression in aleurone cells (Knetsch et al., 1996). In view of such studies, it would be interesting to determine whether the potential 14-3-3 binding site in VP 1 is required for transactivation of the Em gene by VP 1 in a transient assay.

Our results clearly demonstrate that VP 1 can interact with GF14 in yeast and that GF14 is found in the nucleus complexed with Emla, an ABA-VP1 response element in the Em promoter. Because GF14 proteins cannot bind DNA but are part of the Emla complex, our results indicate that GF14 may facilitate the interaction of specific DNA binding proteins and VP1 with Emla through protein-protein interactions, possibly through their $\mathrm{N}$-terminal dimerization domain. Whether and how intermediates in the ABA response pathway interact with these factors are not yet clear (Quatrano et al., 1997).

\section{METHODS}

\section{Two-Hybrid Analysis of Protein-Protein Interactions in Yeast}

Protocols, yeast strains, and plasmid vectors used for the two-hybrid screen are described in Ausubel et al. (1994). The plasmid encoding the VIVIPAROUS1 (VP1) bait was constructed by subcloning the 1.2-kb Xmnl fragment from pAN15 (Hill et al., 1996) into the BamHI (filled-in) sites of $p E G 202$, creating a translational fusion between the LexA DNA binding domain and VP1 (amino acids 190 to 588). The plasmid encoding the nonspecific or control bait was constructed by using polymerase chain reaction amplification of $144 \mathrm{bp}$ of the $C$ terminus of human $\beta 1$-integrin and cloning this fragment into the EcoRINotl sites of pEG202 (C.B. Taylor and P. Nagpal, unpublished results). When the yeast strain EGY 48 was used, both the VP1 and control baits were found to be positive in the repression assay and negative in the activation assay (Ausubel et al., 1994). Vectors without bait (i.e., LexA alone) or with the control bait were not active in any interaction with GF14. However, the control bait was shown to interact with proteins other than GF14 (C.B. Taylor and P. Nagpal, unpublished results).

The cDNA expression library was constructed by mass excising a CDNA expression library from embryogenic rice suspension cells in lambda UNI-ZAP XR, according to the manufacturer's recommendations (Stratagene, La J olla, CA), gel purifying the CDNA inserts, and religating them into the interaction vector pJ G4-5. This library had a complexity of $2 \times 10^{5}$, which was amplified, followed by the isolation of plasmid DNA and transformation into yeast. Plasmids were recovered from yeast showing both phenotypes, as described in Ausubel et al. (1994). Purified plasmids were sequenced and reintroduced into yeast to verify interaction with VP1 bait. Sequences of CDNA inserts in pJ G4-5 were determined using the following primers: forward 5'-TTAACGATACCAGCCTCTTGCTGAGT-3', and reverse 5'-TTGACCAAACCTCTGGCGAAGAAGTC-3'.

The plasmid encoding the interactor protein osGF14b(II) was produced by ligating the Smal-Xhol fragment from pE10-16 into the EcoRI (filled-in)-Xhol sites of pJ G4-5, creating pTS43(2). The plasmid encoding the oSGF14c interactor was constructed by ligating the EcoRI-Xmnl fragment of pE10-15 into the EcoRI/Xhol (filled-in) sites of pJ G4-5, creating pTS52.

\section{Purification of Fusion Proteins and Protein Analysis}

Maltose binding protein (MBP) fusions were expressed and purified as described by Hill et al. (1996). MBP-osGF14a was produced from pAN31 (Hill et al., 1996). MBP-osGF14b(I) was produced from pTS30, which was constructed by cloning the 500-bp EcoRI-HindIII fragment from pE10 into the same sites of pPR997. MBP-osGF14b(II) was produced from pTS51 and was constructed by ligating the 800-bp SmalHindlll from E10-16 into the EcoRI (filled in)-Hindlll sites of pPR997. Protein concentrations were determined using the Bio-Rad protein assay and band intensity of Coomassie Brilliant Blue R 250 -stained gels.

Proteins were solubilized in $1 \times$ SDS sample buffer and separated on $10 \%$ SDS-polyacrylamide gels. Proteins were transferred to nitrocellulose membranes (BA-S; Schleicher $\&$ Schuell) in transfer buffer ( $25 \mathrm{mM}$ Tris, $200 \mathrm{mM}$ glycine, and $20 \%$ methanol) at $100 \mathrm{~V}$ for $1 \mathrm{hr}$ in a mini trans-blot cell (Bio-Rad). Nitrocellulose filters were blocked at room temperature for 1 to $4 \mathrm{hr}$ or overnight at $4^{\circ} \mathrm{C}$ in $1 \times \mathrm{PBS}$ and $8 \%$ nonfat dry milk. Antibodies were diluted in $1 \times$ PBS, $0.05 \%$ Tween 20 , and $0.2 \%$ BSA and incubated with the filters for $1 \mathrm{hr}$ at room temperature, followed by three washes for 5 min each in an excess of $1 \times$ PBS and $0.05 \%$ Tween 20 . Antibody dilutions were as follows: AbFcA (1:1000; gift of P. Aducci [Universita di Roma, Rome, Italy]), MAb GF14(15 and 19) (1:10,000; gift of R. Ferl [University of Florida, Gainsville, FL]), anti-MBP antibody (1:5000; Bio-Rad), anti-mouse conjugated to alkaline phosphatase (1:2500; A-1902; Sigma), and anti-rabbit conjugated to alkaline phosphatase (1:2500; A-7539; Sigma). Filters were developed in a solution containing $50 \mathrm{mM}$ $\mathrm{NaHCO}_{3}, 10 \mathrm{mM} \mathrm{MgCl}, 50 \mathrm{mg} / \mathrm{mL}$ nitro blue tetrazolium, and 150 $\mathrm{mg} / \mathrm{mL}$ 5-bromo-4-chloro-3-indolyl phosphate at room temperature, followed by extensive washing in water. 


\section{Nuclear Extracts and Electrophoretic Mobility Shift Assays}

Nuclear extracts were prepared from embryogenic rice suspension cells and from wild-type and vp1 mutant maize embryos dissected from seeds 20 days after pollination, as described by Guiltinan et al. (1990). Protein concentrations were determined using the Bio-Rad protein assay, according to the manufacturer's protocols. DNA binding reactions and electrophoretic mobility shift assays (EMSAs) were performed as described by Hill et al. (1996), except that $1 \mathrm{mg}$ of nuclear extract, $1 \mathrm{mg}$ poly $(\mathrm{dl}-\mathrm{dC})$, and $2 \mathrm{mg}$ of acetylated BSA were added to each binding reaction. The MAb GF14(19) monoclonal antibody was diluted 1:500, and $4 \mathrm{~mL}$ was added to DNA binding reactions for the supershift experiments. The anti-MBP antibody (New England Biolabs, Beverly, MA) was used undiluted, and $1 \mathrm{~mL}$ was added to the DNA binding reactions.

\section{Protein-Protein Cross-Linking}

MBP fusions were expressed and purified as described in Hill et al. (1996). MBP-cl28 was a product of a Fucus gene (C.B. Taylor, unpublished results), MBP-VP1 was produced from pAN15, MBPEmBP1 from pAN11 (Hill et al., 1996), and MBP-osGF14b(I) from pTS30 (which was constructed by cloning the 500-bp EcoRI-HindIII fragment from $p E 10$ into the same sites of pPR 997). Each fusion protein $(400 \mathrm{ng}$ ) was mixed with MBP-osGF14b(I) (400ng) in $50 \mu \mathrm{L}$ of $1 \times$ PBS in the presence of $2 \mu \mathrm{M}$ dimethyl 3,3'-dithiobispropionimidate (Pierce Chemicals) and incubated on ice for $30 \mathrm{~min}$. The cross-linking reactions were then quenched by the addition of $500 \mu \mathrm{L}$ of Trisbuffered saline and $0.05 \%$ Tween 20 . Proteins were immunoprecipitated using $0.4 \mu \mathrm{L}$ MAb GF14(19) at $4^{\circ} \mathrm{C}$ for $1.5 \mathrm{hr}$ and protein A-Sepharose (Pharmacia). Protein complexes were solubilized in SDS sample buffer, which reduces the cross-linker, separated on a $10 \%$ SDS-polyacrylamide gel, blotted to nitrocellulose membranes (BA-S), and probed with affinity-purified antibodies specific for each fusion protein.

\section{ACKNOWLEDGMENTS}

We thank Drs. Robert J. Ferl and Patrizia Aducci for antibodies to plant 14-3-3 proteins and Drs. Donald R. McCarty and Hirofumi Uchimiya for providing Vp1 and GF14 CDNAs, respectively. We are grateful to Susan Whitfield for her excellent assistance in producing the figures for this manuscript and members of the Quatrano laboratory, especially Drs. Crispin B. Taylor and Punita Nagpal, for their helpful discussions and encouragement throughout this work. A.H. was the recipient of National Institutes of Health Research Service Award Grant No. GM 13588; the research was supported by Grant No. GM 44288 from the National Institutes of Health to R.S.Q.

Received December 18, 1997; accepted March 9, 1998.

\section{REFERENCES}

Aitken, A. (1996). 14-3-3 and its possible role in co-ordinating multiple signaling pathways. Trends Cell Biol. 6, 341-347.
Ausubel, F.M., Brent, R., Kingston, R.E., Moore, D.D., Seidman, J .G., Smith, J .A., and Struhl, K., eds (1994). Protocols in Molecular Biology (New York: J ohn Wiley and Sons), Chapter 20, pp. 1.1-1.20.

Bobb, A.J ., Eiben, H.G., and Bustos, M.M. (1995). PvAlf, an embryo-specific acidic transcriptional activator, enhances gene expression from phaseolin and phytohemmagglutinin promoters. Plant J . 8, 331-343.

Chen, Z., Fu, H., Liu, D., Chang, P.F., Narasimhan, M., Ferl, R., Hasegawa, P.M., and Bressan, R.A. (1994). A NaCl-regulated plant gene encoding a brain protein homology that activates ADP ribosyltransferase and inhibits protein kinase C. Plant J . 6, 729-740.

Daugherty, C.J ., Rooney, M.F., Miller, P.W., and Ferl, R.J . (1996). Molecular organization and tissue-specific expression of an Arabidopsis 14-3-3 gene. Plant Cell 8, 1239-1248.

de Vetten, N.C., Lu, G., and Ferl, R.J . (1992). A maize protein associated with the $\mathrm{G}$ box binding complex has homology to brain regulatory proteins. Plant Cell 4, 1295-1307.

Estojak, J ., Brent, R., and Golemis, E.A. (1995). Correlation of twohybrid affinity data with in vitro measurements. Mol. Cell. Biol. 15, 5820-5829.

Ferl, R.J . (1996). 14-3-3 proteins and signal transduction. Annu. Rev. Plant Physiol. Plant Mol. Biol. 47, 49-73.

Finkelstein, R.R. (1993). Abscisic acid-insensitive mutations provide evidence for stage-specific signal pathways regulating expression of an Arabidopsis late embryo-abundant (lea) gene. Mol. Gen. Genet. 238, 401-408.

Giraudat, J., Hauge, B.M., Valon, C., Smalle, J., Parcy, F., and Goodman, H.M. (1992). Isolation of the Arabidopsis ABI3 gene by positional cloning. Plant Cell 4, 1251-1261.

Giraudat, J ., Parcy, F., Bertauche, N., Gosti, F., Leung, J ., Morris, P.-C., Bouvier-Durand, M., and Vartanian, N. (1994). Current advances in abscisic acid action and signaling. Plant Mol. Biol. 26, 1557-1577.

Goodrich, J .A., Cutler, G., and Tjian, R. (1996). Contacts in context: Promoter specificity and macromolecular interactions in transcription. Cell 84, 825-830.

Guiltinan, M.J ., Marcotte, W.R., J r., and Quatrano, R.S. (1990). A plant leucine zipper protein that recognizes an abscisic acid response element. Science 250, 267-271.

Hattori, T., Terada, T., and Hamasuna, S. (1994). Sequence and functional analyses of the rice gene homologous to the maize Vp1. Plant Mol. Biol. 24, 805-810.

Hattori, T., Terada, T., and Hamasuna, S. (1995). Regulation of the Osem gene by abscisic acid and the transcriptional activator VP 1: Analysis of cis-acting promoter elements required for regulation by abscisic acid and VP1. Plant J . 7, 913-925.

Hill, A., Nantel, A., Rock, C.D., and Quatrano, R.S. (1996). A conserved domain of the viviparous-1 gene product enhances the DNA binding activity of the bZip protein EmBP-1 and other transcription factors. J. Biol. Chem. 271, 3366-3374.

Huber, S.C., Bachmann, M., and Huber, J .L. (1996). Post-translational regulation of nitrate reductase activity: $\mathrm{A}$ role for $\mathrm{Ca}^{2+}$ and 14-3-3 proteins. Trends Plant Sci. 1, 432-438.

Izawa, T., Foster, R., and Chua, N.-H. (1993). Plant bZIP protein DNA binding specificity. J . Mol. Biol. 230, 1131-1144. 
Jahn, T., Fuglsang, A.T., Olsson, A., Brüntrup, I.M., Collinge, D.B., Volkmann, D., Sommarin, M., Palmgren, M.G., and Larsson, C. (1997). The 14-3-3 protein interacts directly with the $\mathrm{C}$-terminal region of the plant plasma membrane $\mathrm{H}^{+}$-ATPase. Plant Cell 9, 1805-1814.

J ones, H.D., Peters, N.C.B., and Holdsworth, M.J . (1997). Genotype and environment interact to control dormancy and differential expression of the VIVIPAROUS1 homologue in embryos of Avena fatua. Plant J . 12, 911-920.

Kidou, S., Umeda, M., Kato, A., and Uchimiya, H. (1993). Isolation and characterization of a rice cDNA similar to the bovine brainspecific 14-3-3 protein gene. Plant Mol. Biol. 21, 191-194.

Knetsch, M.L.W., Wang, M., Snaar-Jagalska, B.E., and Heimovaara-Dijkstra, S. (1996). Abscisic acid induces mitogenactivated protein kinase activation in barley aleurone protoplasts. Plant Cell 8, 1061-1067.

Leung, J., Bouvier-Durand, M., Morris, P.-C., Guerrier, D., Chefdor, F., and Giraudat, J . (1994). Arabidopsis ABA-response gene $A B \mid 1$ : Features of a calcium-modulated protein phosphatase. Science 264, 1448-1452.

Leung, J., Merlot, S., and Giraudat, J . (1997). The Arabidopsis ABSCISIC ACID-INSENSITIVE2 (ABI2) and ABI1 genes encode homologous protein phosphatases $2 \mathrm{C}$ involved in abscisic acid signal transduction. Plant Cell 9, 759-771.

Liu, D., Bienkowska, J., Petssa, C., Collier, R.J., Fu, H., and Liddington, R. (1995). Crystal structure of the zeta isoform of the 14-3-3 protein. Nature 376, 191-194.

Lu, G., DeLisle, A.J ., de Vetten, N.C., and Ferl, R.J . (1992). Brain proteins in plants: An Arabidopsis homolog to neurotransmitter pathway activators is part of a DNA binding complex. Proc. Natl. Acad. Sci. USA 89, 11490-11494.

Lu, G., Sehnke, P.C., and Ferl, R.J . (1994). Phosphorylation and calcium binding properties of an Arabidopsis GF14 brain protein homolog. Plant Cell 6, 501-510.

Marcotte, W.R., J r., Bayley, C.C., and Quatrano, R.S. (1988). Regulation of a wheat promoter by abscisic acid in rice protoplasts. Nature 335, 454-457.

Marcotte, W.R., J r., Russell, S.H., and Quatrano, R.S. (1989). Abscisic acid-responsive sequences from the Em gene of wheat. Plant Cell 1, 969-976.

Marra, M., Fullone, M.R., Fogliano, V., Pen, J ., Mattei, M., Masi, S., and Aducci, P. (1994). The 30-kilodalton protein present in purified fusicoccin receptor preparations is a 14-3-3-like protein. Plant Physiol. 106, 1497-1501.

McCarty, D.R. (1995). Genetic control and integration of maturation and germination pathways in seed development. Annu. Rev. Plant Physiol. Plant Mol. Biol. 46, 71-93.

McCarty, D.R., Carson, C.B., Lazar, M., and Simonds, S.C. (1989a). Transposable element induced mutations of the viviparous-1 gene of maize. Dev. Genet. 10, 473-481.

McCarty, D.R., Carson, C.B., Stinard, P.S., and Robertson, D.S. (1989b). Molecular analysis of viviparous-1: An abscisic acidinsensitive mutant of maize. Plant Cell 1, 523-532.

McCarty, D.R., Hattori, T., Carson, C.B., Vasil, V., Lazar, M., and Vasil, I.K. (1991). The viviparous1 developmental gene of maize encodes a novel transcriptional activator. Cell 66, 895-905.
Meyer, K., Leube, M.P., and Grill, E. (1994). A protein phosphatase $2 \mathrm{C}$ involved in $\mathrm{ABA}$ signal transduction in Arabidopsis thaliana. Science 264, 1452-1455.

Muslin, A.J ., Tanner, J.W., Allen, P.M., and Shaw, A.S. (1996). Interaction of 14-3-3 with signaling proteins is mediated by the recognition of phosphoserine. Cell 84, 889-897.

Nambara, E., Naito, S., and McCourt, P. (1992). A mutant of Arabidopsis which is defective in seed development and storage protein accumulation is a new abi3 allele. Plant J . 2, 435-441.

Nantel, A., and Quatrano, R.S. (1996). Characterization of three rice bZIP factors, including two inhibitors of EmBP-1 DNA-binding activity. J . Biol. Chem. 271, 31296-31305.

Parcy, F., Valon, C., Raynal, M., Gaubier-Comella, P., Delseny, M., and Giraudat, J . (1994). Regulation of gene expression programs during Arabidopsis seed development: Roles of the $A B I 3$ locus and of endogenous abscisic acid. Plant Cell 6, 1567-1582.

Pei, Z.-M., Kuchitsu, K., Ward, J.M., Schwarz, M., and Schroeder, J .I. (1997). Differential abscisic acid regulation of guard cell slow anion channels in Arabidopsis wild-type and abi1 and abi2 mutants. Plant Cell 9, 409-423.

Quatrano, R.S., Bartels, D., Ho, T.-H.D., and Pages, M. (1997). New insights into ABA-mediated processes. Plant Cell 9, 470-475.

Razik, M.A., and Quatrano, R.S. (1997). Effect of the nuclear factors EmBP1 and Viviparous1 on the transcription of the Em gene in HeLa nuclear extracts. Plant Cell 9, 1791-1803.

Rock, C.D., and Quatrano, R.S. (1994). Insensitivity is in the genes. Curr. Biol. 4, 1013-1015.

Rock, C.D., and Quatrano, R.S. (1995). The role of hormones during seed development. In Plant Hormones: Physiology, Biochemistry and Molecular Biology, P.J. Davies, ed (Dordrecht, The Netherlands: Kluwer Academic Publishers), pp. 671-697.

Schindler, U., Menkens, A.E., Beckmann, H., Ecker, J.R., and Cashmore, A.R. (1992). Heterodimerization between light-regulated and ubiquitously expressed Arabidopsis thaliana GBF bZIP proteins. EMBO J . 4, 1261-1273.

Schultz, T., and Quatrano, R.S. (1997). Characterization and expression of a rice RAD23 gene. Plant Mol. Biol. 34, 557-562.

Strynadka, N.C.J ., and J ames, M.N.G. (1989). Crystal structures of the helix-loop-helix calcium-binding proteins. Annu. Rev. Biochem. 58, 951-998.

Suzuki, M., Kao, C.Y., and McCarty, D.R. (1997). The conserved B3 domain of VIVIPAROUS1 has a cooperative DNA binding activity. Plant Cell 9, 799-807.

Vasil, V., Marcotte, W.R., J r., Rosenkrans, L., Cocciolone, S.M., Vasil, I.K., Quatrano, R.S., and McCarty, D.R. (1995). Overlap of viviparous (VP1) and abscisic acid response elements in the Em promoter: $\mathrm{G}$ box elements are sufficient but not necessary for VP 1 transactivation. Plant Cell 7, 1511-1518.

Wu, K., Rooney, M.F., and Ferl, R.J . (1997). The Arabidopsis 14-3-3 multigene family. Plant Physiol. 114, 1421-1431.

Zeevart, J .A.D., and Creelman, R.A. (1988). Metabolism and physiology of abscisic acid. Annu. Rev. Plant Physiol. Plant Mol. Biol. 39, 439-473. 
14-3-3 Proteins Are Part of an Abscisic Acid-VIVIPAROUS1 (VP1) Response Complex in the Em

Thomas F. Schultz, Joaquin Medina, Alison Hill and Ralph S. Quatrano

Plant Cell 1998;10;837-847

DOI 10.1105/tpc.10.5.837

This information is current as of July 22, 2020

$\begin{array}{ll}\text { References } & \text { This article cites } 49 \text { articles, 24 of which can be accessed free at: } \\ & \text { /content/10/5/837.full.html\#ref-list-1 } \\ \text { Permissions } & \text { https://www.copyright.com/ccc/openurl.do?sid=pd_hw1532298X\&issn=1532298X\&WT.mc_id=pd_hw1532298X } \\ \text { eTOCs } & \begin{array}{l}\text { Sign up for eTOCs at: } \\ \text { http://www.plantcell.org/cgi/alerts/ctmain }\end{array} \\ \text { CiteTrack Alerts } & \begin{array}{l}\text { Sign up for CiteTrack Alerts at: } \\ \text { http://www.plantcell.org/cgi/alerts/ctmain }\end{array} \\ \text { Subscription Information } & \begin{array}{l}\text { Subscription Information for The Plant Cell and Plant Physiology is available at: } \\ \text { http://www.aspb.org/publications/subscriptions.cfm }\end{array}\end{array}$

